\title{
Initial state angular asymmetries in high energy p+A collisions: spontaneous breaking of rotational symmetry by a color electric field and C-odd fluctuations
}

\author{
Adrian Dumitru* \\ Department of Natural Sciences, Baruch College, CUNY, \\ 17 Lexington Avenue, New York, NY 10010, USA \\ Andre V. Giannini ${ }^{\dagger}$ \\ Instituto de Física, Universidade de São Paulo, C.P. 66318, 05315-970 São Paulo, SP, Brazil \\ Department of Natural Sciences, Baruch College, CUNY, \\ 17 Lexington Avenue, New York, NY 10010, USA
}

\begin{abstract}
We present a simple model for generating initial-state azimuthal asymmetries in pA collisions from dipole scattering on an anisotropic dense target. Parity even angular harmonics arise from the C-even real part of the dipole S-matrix which spontaneously breaks rotational symmetry in two dimensions due to a condensate for the color electric field. This leads to an angular correlation with the direction of $\vec{E}$. Parity odd harmonics are generated by the C-odd imaginary part (odderon) due to coupling to coherent target fluctuations which again break rotational invariance. We perform a first qualitative extraction of the amplitude and cutoff of C-odd fluctuations in the dense target.
\end{abstract}

\section{INTRODUCTION}

Large azimuthal asymmetries have been observed in $\mathrm{p}+\mathrm{Pb}$ collisions at the LHC by the ALICE [1], ATLAS $[2,3]$ and CMS [4] collaborations. The PHENIX collaboration has also reported similar asymmetries from $\mathrm{d}+\mathrm{Au}$ collisions at RHIC [5]. These asymmetries are usually measured via multi-particle angular correlations (see below) and were found to extend over a long range in rapidity. By causality, the correlations should therefore originate from the earliest times of the collision [6]. Recently, PHENIX has shown that the $v_{2}$ quadrupole moment in the central region of central $\mathrm{d}+\mathrm{Au}$ collisions can also be observed in terms of a correlation of particles with a global "event plane" [7].

Azimuthal harmonics $v_{n}=\langle\cos n \phi\rangle$ defined as $\cos n \phi$ moments of the single-inclusive distribution require spontaneous breaking of rotational symmetry in the transverse plane, defining the event plane. In a classical impact parameter picture of a binary collision such a preferred direction is provided by the impact parameter vector. For single-spin asymmetries in collisions of polarized protons with a heavy ion, the preferred direction is due to the polarization of the projectile [8]. Here, we explore the scenario that rotational symmetry is broken by a condensate for the electric field $\vec{E}$ in the target [9], leading to $v_{2 n} \neq 0$; and by spontaneous C-odd fluctuations in the target [10], leading to $v_{2 n+1} \neq 0$.

The quadrupole moment $v_{2}$ in the initial state of high-energy collisions has been first calculated by Kovchegov and Tuchin long ago [11]. Non-zero $v_{2}$ emerges due to the fact that the two-gluon production cross section at relative angle $\phi_{1}-\phi_{2} \sim 0, \pi$ is enhanced, while it is suppressed for $\phi_{1}-\phi_{2} \sim \pm \pi / 2$. Hence, the two-particle cumulant [12]

$$
v_{2}^{2}\{2\}=\left\langle e^{2 i\left(\phi_{1}-\phi_{2}\right)}\right\rangle
$$

does not vanish. A more recent analysis which also addresses higher moments $v_{n}^{2}\{2\}=\left\langle e^{i n\left(\phi_{1}-\phi_{2}\right)}\right\rangle$ of gluons emitted off the large- $x$ sources has been presented by Gyulassy et al. [13].

However, $v_{2}\{2\}$ fluctuates between events due to the presence of "random" sources of two-particle correlations. These contributions, if described by a Bessel-Gaussian distribution, are suppressed by higher-order multi-particle cumulants [12] such as

$$
v_{2}^{4}\{4\}=2\left\langle e^{2 i\left(\phi_{1}-\phi_{3}\right)}\right\rangle\left\langle e^{2 i\left(\phi_{2}-\phi_{4}\right)}\right\rangle-\left\langle e^{2 i\left(\phi_{1}+\phi_{2}-\phi_{3}-\phi_{4}\right)}\right\rangle .
$$

The fact that $v_{2}\{2\} \neq 0$ or $v_{2}\{4\} \neq 0$ does not imply that particles are correlated with a global "event plane" (i.e. spontaneous breaking of rotational symmetry). For a finite number of particles or sources, multi-particle $v_{2}$

\footnotetext{
*Electronic address: adrian.dumitru@baruch.cuny.edu

${ }^{\dagger}$ Electronic address: avgiannini@usp.br
} 
correlations can also emerge from fluctuations [14]. In this paper, however, we explore whether the $p_{T}$ dependence of $v_{2}\left(p_{T}\right)$ could be consistent with spontaneous breaking of rotational symmetry due to a condensate for the (color) electric field.

Kovner and Lublinsky have argued the formation of an electric field condensate at the saturation scale $Q_{s}$ in large, boosted nuclei [9]. It is supposed to arise from fluctuations in the impact parameter dependent small- $x$ evolution in a system with a correlation length of order $1 / Q_{s}$. If there is a finite correlation length of order $1 / Q_{s}$ it appears reasonable that over such distance scales the $\vec{E}$ field might point in a fixed (but random) direction. Such an $\vec{E}$-field condensate spontaneously breaks rotational symmetry about the beam axis since an incoming charge is "deflected" anisotropically. While the direction of $\vec{E}$ in a particular event is of course random, the azimuthal distribution of the single inclusive parton cross section with respect to this preferred direction is not uniform ${ }^{1}$. The condensate provides a preferred direction for any particle in an event scattering off that particular $\vec{E}$ field. Here, one of our main goals is to evaluate the $p_{T}$ dependence of $v_{2}$ and $v_{4}$ for a $\vec{r} \cdot \vec{E}$ dipole - electric field interaction. While we focus on angular harmonics at a fixed rapidity here, the correlations in fact extend over a long range in rapidity due to the approximate boost invariance of the target fields [15].

We do not account for fluctuation induced $v_{2}$ (indeed, our $v_{2 n}$ vanish in the absence of the condensate) and so our results are more closely related to $v_{2}\{4\}$ rather than $v_{2}\{2\} .{ }^{2}$ Also, we recall that at leading order in the number of colors $N_{c}$, in this approach the $n$-particle distribution factorizes into $n$ single-particle distributions, each of which exhibits an angular correlation with the global "event plane" defined by the $\vec{E}$-field condensate [15]. Connected two-particle production diagrams $[6,16]$ appear at relative order $\sim 1 / N_{c}^{2}$ and provide corrections to factorization of angular correlations.

Odd azimuthal harmonics $v_{2 n+1}$ may also be induced by C-odd fluctuations [17]. These moments emerge from parity odd (under $\phi \rightarrow \phi+\pi$ ) contributions to the single-inclusive distribution, see below. In the dipole picture, they are related to the C-odd imaginary part of the dipole S-matrix [10], just like single-spin asymmetries in collisions of polarized protons on heavy-ion targets [8, 18]. Our second main goal is to provide some first model studies of $v_{1}$ and $v_{3}$ (without final state interactions) and to extract an amplitude and scale for C-odd fluctuations. Just like our calculation of even harmonics $v_{2 n}$, we shall assume that all wave vectors of C-odd fluctuations point in the same direction and so provide a preferred "global" direction for $v_{2 n+1}$ which breaks rotational symmetry spontaneously.

\section{A. Basic setup}

We work in the so-called "hybrid formalism" where the proton projectile is treated as a beam of collinear partons with a large light-cone momentum $p^{-}$which scatter off the field of the target. To leading order in $p_{\perp} / p^{-}$projectile partons propagate on eikonal trajectories and the amplitude corresponding to elastic scattering from momentum $p$ to $q$ is $[19]$

$$
\begin{aligned}
\langle\text { out, } q| \text { in, } p\rangle & \equiv \bar{u}(q) \tau(q, p) u(p) \\
\tau(q, p) & =2 \pi \delta\left(p^{-}-q^{-}\right) \gamma^{-} \int d^{2} \vec{x}[V(\vec{x})-1] e^{i(\vec{p}-\vec{q}) \cdot \vec{x}}
\end{aligned}
$$

Here,

$$
V(\vec{x})=\mathcal{P} \exp \left[i g \int d x^{-} A^{+}\left(x^{-}, \vec{x}\right)\right]
$$

is a Wilson line along the light cone. Squaring the amplitude gives the scattering cross section [20]

$$
\frac{d \sigma}{d^{2} b d^{2} k}=\frac{d N}{d^{2} k}=\frac{1}{(2 \pi)^{2}} \int d^{2} r e^{-i \vec{k} \cdot \vec{r}}\left(\left\langle\frac{1}{N_{c}} \operatorname{tr}\left(W(\vec{r}, \vec{b})-V(\vec{b}-\vec{r} / 2)-V^{\dagger}(\vec{b}+\vec{r} / 2)\right)\right\rangle+1\right) .
$$

Here, $\vec{b}$ denotes the impact parameter of the collision and $W(\vec{r}, \vec{b})$ is a light-like Wilson loop of width given by $r=|\vec{r}|$. In covariant gauge $W(\vec{r}, \vec{b})=V^{\dagger}(\vec{b}+\vec{r} / 2) V(\vec{b}-\vec{r} / 2)$, commonly referred to as the dipole unintegrated gluon

\footnotetext{
${ }^{1}$ In this sense, $v_{2}$ in $\mathrm{p}+\mathrm{A}$ collisions from a vector target electric field is analogous to the "standard" $v_{2}$ from non-central A+A collisions where rotational symmetry is spontaneously broken by a non-zero impact parameter $\vec{b}$, which points in a random direction.

${ }^{2}$ We should emphasize again that for a finite number of particles, fluctuations can induce two- as well as multi-particle correlations in coordinate space [14] which might then generate correlations in momentum space in the final state; this is to be distinguished from the effect studied here.
} 
distribution [21]. The size of the dipole is given by the shift of the transverse coordinate of the eikonal quark line from the amplitude to the complex conjugate amplitude, respectively. The expression in parenthesis corresponds to the S-matrix for a dipole of size and orientation given by $\vec{r}$.

Averaging over a C-even ensemble of target fields in eq. (6) provides the real part $D(\vec{r})$ of the dipole S-matrix which is even under $\vec{r} \rightarrow-\vec{r}$, see below. On the other hand, C-odd fluctuations provide an expectation value for the "odderon" $\operatorname{Im} S=O(\vec{r})$ which is odd under $\vec{r} \rightarrow-\vec{r}$. Eq. (6) can be turned into a physical $p A \rightarrow h+X$ single inclusive cross section for production of a hadron of type $h$ via a convolution with a proton-parton distribution and a corresponding $q \rightarrow h$ fragmentation function [22-24]. The present paper does not aim at a quantitative comparison to the data and so we presently drop these convolutions with the parton distribution and fragmentation functions. Our more modest goal is to obtain some basic understanding of the behavior of the azimuthal harmonics $v_{n}$ from eq. (6).

We should stress that while eq. (6) does resum coherent multiple scattering of a projectile parton with the target field, it intrinsically assumes that the projectile is dilute and that non-linear (high density) effects in the proton can be neglected. This may not be a good representation of high multiplicity $\mathrm{p}+\mathrm{A}$ collisions at LHC energies (except towards the fragmentation region of the proton [20]). Small- $x$ evolution effects of the proton can in principle be treated, see e.g. refs. [16] specifically for studies of the "ridge" resp. of $v_{2}$ in the fluctuation dominated regime. A related issue is that non-trivial field configurations such as semi-hard "strings" can form in collisions of dense sheets of color charge, and their decay may also induce angular correlations [25]. However, given the relative simplicity with which we can incorporate an electric field condensate in eq. (6) we find it interesting to explore the angular distribution it predicts, and in particular to show how parity odd angular moments of the single-inclusive distribution are generated by fluctuations of the target field.

\section{TRANSVERSE MOMENTUM DISTRIBUTION OF SCATTERED QUARKS}

The transverse momentum distribution of quarks scattered off the target can now be written as ${ }^{3}$

$$
\begin{aligned}
(2 \pi)^{2} \frac{d N}{k d k d \phi_{k}} & =\int d^{2} r e^{-i \vec{k} \cdot \vec{r}} S(\vec{r}) \\
& =\int d r r d \phi_{r} e^{-i k r \cos \left(\phi_{k}-\phi_{r}\right)} S\left(r, \phi_{r}\right),
\end{aligned}
$$

with $S(\vec{r})$ the S-matrix of a dipole of size and orientation given by $\vec{r}$. The transverse momentum distribution is a real function, hence

$$
S\left(r, \phi_{r}\right)=S^{*}\left(r, \phi_{r}+\pi\right)
$$

In particular, the real and imaginary parts of $S(\vec{r})$ satisfy

$$
\begin{aligned}
S\left(r, \phi_{r}\right) & =D\left(r, \phi_{r}\right)+i O\left(r, \phi_{r}\right), \\
D\left(r, \phi_{r}\right) & =D\left(r, \phi_{r}+\pi\right), \\
O\left(r, \phi_{r}\right) & =-O\left(r, \phi_{r}+\pi\right) .
\end{aligned}
$$

Thus, the real part $D(\vec{r})$ is even under $\phi_{r} \rightarrow \phi_{r}+\pi$ (i.e. $\vec{r} \rightarrow-\vec{r}$ ) while $O(\vec{r})$ is odd.

The S-matrix for a dipole in the adjoint representation on the other hand is real. Written in terms of the real and imaginary parts of the fundamental representation from eq. (10) it is

$$
S_{\text {adj }}(\vec{r})=\frac{N_{c}^{2}\left[D^{2}(\vec{r})+O^{2}(\vec{r})\right]-1}{N_{c}^{2}-1} \rightarrow D^{2}(\vec{r})+O^{2}(\vec{r})
$$

where the right most expression applies in the large- $N_{c}$ limit. Clearly $S_{\text {adj }}(\vec{r})=S_{\text {adj }}(-\vec{r})$ and thus can only generate non-zero $v_{2 n}$; this has been pointed out before in ref. [15].

\footnotetext{
${ }^{3}$ Eq. (7) includes the "no scattering" contribution for transverse momentum exchange $k=0$. It plays no role in our subsequent analysis since we are interested in finite $k$ only.
} 


\section{AZIMUTHAL HARMONICS $v_{n}$}

We can define various asymmetry moments $v_{n}$ through

$$
v_{n}\left(k_{T}\right)=\left\langle\cos n \phi_{k}\right\rangle=\frac{1}{\mathcal{N}} \int \frac{d \phi_{k}}{2 \pi} \cos \left(n \phi_{k}\right) \frac{d N}{d y k_{T} d k_{T} d \phi_{k}},
$$

with

$$
\mathcal{N}=\int \frac{d \phi_{k}}{2 \pi} \frac{d N}{k_{T} d k_{T} d \phi_{k}}=\frac{1}{\pi} \frac{d N}{d k_{T}^{2}}
$$

Even (odd) moments have positive (negative) parity:

$$
\begin{aligned}
\left\langle\cos 2 n \phi_{k}\right\rangle & =+\left\langle\cos 2 n\left(\phi_{k}+\pi\right)\right\rangle, \\
\left\langle\cos (2 n+1) \phi_{k}\right\rangle & =-\left\langle\cos (2 n+1)\left(\phi_{k}+\pi\right)\right\rangle .
\end{aligned}
$$

If the scattering amplitude $S\left(r, \phi_{r}\right)$ is independent of the orientation of the dipole then all $v_{n}=0$. An angular dependence of its real part $D\left(r, \phi_{r}\right)$ gives rise to non-zero parity even moments $v_{2 n}$; an angular dependence of $O\left(r, \phi_{r}\right)$ produces odd moments $v_{2 n+1}$.

In the following sections we employ simple schematic models for the dipole S-matrix to work out qualitative features of the azimuthal moments $v_{n}\left(k_{T}\right)$.

\section{MODELS FOR THE REAL PART OF THE DIPOLE SCATTERING AMPLITUDE}

\section{A. Quasi-classical dipole model}

In the classical McLerran-Venugopalan model of Gaussian color charge fluctuations the real part of the dipole scattering amplitude is given by [26]

$$
D(r)=e^{-\frac{1}{4} r^{2} Q_{s}^{2} \log \frac{1}{\Lambda r}} .
$$

To arrive at this expression one has averaged over all configurations of the target and so $D(\vec{r})$ is rotationally symmetric. With this rotationally symmetric dipole the transverse momentum distribution at $k_{T} \gg Q_{s}$ becomes

$$
\frac{d N}{k_{T} d k_{T} d \phi_{k}}=\frac{1}{2 \pi} \frac{Q_{s}^{2}}{k_{T}^{4}}+\cdots
$$

Following Kovner and Lublinsky [9] we instead consider an average over the color charge configurations of the target at fixed relative angle of dipole and target field. That is, local rotational symmetry in the transverse plane is spontaneously broken in a particular event by the direction of $E^{i}=F^{+i}$ within the domain. The presence of such a condensate has been argued to provide a possible explanation for the large $v_{2}\{4\}$ observed in high multiplicity $\mathrm{p}+\mathrm{Pb}$ collisions at the LHC, see section 3 in ref. [27].

We perform a global rotation of the event such that $\vec{E}=(E, 0)$ points in the $\vec{x}$-direction ${ }^{4}$. We then have $(\vec{r} \cdot \vec{E})^{2}=$ $E^{2} r^{2} \cos ^{2} \phi_{r}$. Hence

$$
D(\vec{r})=e^{-\frac{1}{4} r^{2} Q_{s}^{2}\left(1-\mathcal{A}+2 \mathcal{A} \cos ^{2} \phi_{r}\right) \log \frac{1}{\Lambda r}},
$$

where $\mathcal{A}$ determines the degree of polarization of the target field, with $\mathcal{A}=1$ corresponding to perfect polarization while $\mathcal{A}=0$ leads back to the unpolarized target from eq. (18). $D(\vec{r})$ clearly satisfies (11) from above, i.e. it has even parity under $\vec{r} \rightarrow-\vec{r}$, and so all $v_{2 n+1}=0$. Note that if

$$
\frac{g^{2}}{N_{c}} \sum_{a, b}\left\langle\vec{r} \cdot \vec{E}^{a} \vec{r} \cdot \vec{E}^{b}\right\rangle \sim r^{2} Q_{s}^{2}\left(1-\mathcal{A}+2 \mathcal{A} \cos ^{2} \phi_{r}\right)
$$

\footnotetext{
4 This is according to the convention that the resulting transverse momentum distribution is symmetric under $\phi_{k} \rightarrow-\phi_{k}$, and that $v_{2} \equiv\left\langle\cos 2 \phi_{k}\right\rangle$ is maximized.
} 
with the same angle $\phi_{r}$ for all color channels then $\mathcal{A}=\mathcal{O}(1)$. On the other hand, if the direction of $\vec{E}^{a}$ in different color channels fluctuates independently then $\mathcal{A}$ is suppressed by the appropriate power of $N_{c}$.

We emphasize that in (20) we have not explicitly averaged over multiple domains of $\vec{E}$ as described in ref. [9] but in essence assume scattering off a single $\vec{E}$ domain with effective polarization $\mathcal{A}$. We present a simple domain model in section VIB.

The transverse momentum distribution of quarks scattered to $k_{T} \gg Q_{s}$ now is

$$
\frac{d N}{k_{T} d k_{T} d \phi_{k}}=\frac{1}{2 \pi} \frac{Q_{s}^{2}}{k_{T}^{4}}\left[1-2 \mathcal{A}\left(k_{T}\right)+4 \mathcal{A}\left(k_{T}\right) \cos ^{2} \phi_{k}\right] .
$$

Because of the absence of non-linear effects for the classical dipole $(20)$ at high $k_{T}$, the angular average $\left(\left\langle\cos ^{2} \phi_{k}\right\rangle=\right.$ 1/2) leads back to the transverse momentum distribution for an unpolarized target from eq. (19).

The angular distribution (22) leads to

$$
v_{2}\left(k_{T}\right) \equiv\left\langle\cos 2 \phi_{k}\right\rangle=\mathcal{A}\left(k_{T}\right)
$$

so that $v_{2}\left(k_{T}\right)$ is related to the target field polarization at the scale $k_{T}$, with all other $v_{n \neq 2}=0$. One may expect that beyond the saturation scale $\mathcal{A}\left(k_{T}\right)$ could decrease with increasing transverse momentum since the short wavelength modes of $\vec{E}$ should have random orientations. In our numerical estimates below for simplicity we shall assume $\mathcal{A}=$ const.

We can also obtain the Fourier transform of (20) at $k_{T} \ll Q_{s}$. Here, the maximal dipole size $r$ is not set to $\sim 1 / k_{T}$ by the Fourier phase but to $\sim 1 / Q_{s}$ by saturation. The transverse momentum distribution becomes

$$
\frac{d N}{k_{T} d k_{T} d \phi_{k}}=\frac{1}{\pi Q_{s}^{2} \log Q_{s} / \Lambda} \frac{1}{\sqrt{1-\mathcal{A}^{2}}} \exp \left[-\frac{k_{T}^{2}}{Q_{s}^{2} \log Q_{s} / \Lambda}\left(\frac{\cos ^{2} \phi_{k}}{1+\mathcal{A}}+\frac{\sin ^{2} \phi_{k}}{1-\mathcal{A}}\right)\right] .
$$

To obtain the normalization factor we average over $\phi_{k}$ :

$$
\frac{1}{\pi} \frac{d N}{d k_{T}^{2}}=\frac{1}{\pi Q_{s}^{2} \log Q_{s} / \Lambda} \frac{1}{\sqrt{1-\mathcal{A}^{2}}} I_{0}\left(\frac{\mathcal{A}}{1-\mathcal{A}^{2}} \frac{k_{T}^{2}}{Q_{s}^{2} \log Q_{s} / \Lambda}\right) \exp \left[-\frac{k_{T}^{2}}{Q_{s}^{2} \log Q_{s} / \Lambda} \frac{1}{1-\mathcal{A}^{2}}\right] .
$$

Hence, the normalized angular distribution is given by

$$
\frac{1}{I_{0}\left(\frac{\mathcal{A}}{1-\mathcal{A}^{2}} \frac{k_{T}^{2}}{Q_{s}^{2} \log Q_{s} / \Lambda}\right)} \exp \left[\frac{k_{T}^{2}}{Q_{s}^{2} \log Q_{s} / \Lambda} \frac{\mathcal{A}}{1-\mathcal{A}^{2}}\left(\cos ^{2} \phi_{k}-\sin ^{2} \phi_{k}\right)\right]
$$

Here, the polarization amplitude $\mathcal{A}=\mathcal{A}\left(Q_{s}\right)$ is measured at the scale $Q_{s}$ and is therefore independent of $k_{T}$. Eq. $(26)$ leads to

$$
\begin{aligned}
& v_{2}\left(k_{T}\right)=\frac{I_{1}\left(\frac{\mathcal{A}}{1-\mathcal{A}^{2}} \frac{k_{T}^{2}}{Q_{s}^{2} \log Q_{s} / \Lambda}\right)}{I_{0}\left(\frac{\mathcal{A}}{1-\mathcal{A}^{2}} \frac{k_{T}^{2}}{Q_{s}^{2} \log Q_{s} / \Lambda}\right)} \simeq \frac{\mathcal{A}}{2} \frac{k_{T}^{2}}{Q_{s}^{2} \log Q_{s} / \Lambda} \\
& v_{4}\left(k_{T}\right)=\frac{I_{2}\left(\frac{\mathcal{A}}{1-\mathcal{A}^{2}} \frac{k_{T}^{2}}{Q_{s}^{2} \log Q_{s} / \Lambda}\right)}{I_{0}\left(\frac{\mathcal{A}}{1-\mathcal{A}^{2}} \frac{k_{T}^{2}}{Q_{s}^{2} \log Q_{s} / \Lambda}\right)} \simeq \frac{\mathcal{A}^{2}}{8}\left(\frac{k_{T}^{2}}{Q_{s}^{2} \log Q_{s} / \Lambda}\right)^{2} .
\end{aligned}
$$

The expressions on the right hand side apply when the polarization amplitude of the target field $\mathcal{A} \ll 1$. Thus, $v_{4}\left(k_{T}\right)$ is not zero in the saturation regime though smaller than $v_{2}\left(k_{T}\right)$ by one additional power of $\mathcal{A}$ and of $k_{T}^{2} / Q_{s}^{2}$.

\section{B. Dipole with anomalous dimension}

The dipole aquires an anomalous dimension $\gamma<1$ upon resummation of quantum fluctuations in the small- $x$ regime:

$$
D(\vec{r})=e^{-\left(\frac{1}{4} r^{2} Q_{s}^{2}\left(1-\mathcal{A}+2 \mathcal{A} \cos ^{2} \phi_{r}\right)\right)^{\gamma}} .
$$

(Logarithms of $1 / r$ do not affect the transverse momentum distribution qualitatively when $\gamma<1$.) 
In this subsection we discuss the corresponding angular distribution and its moments $v_{n}$. We shall restrict to $\gamma=1 / 2$ in order to derive simple analytical expressions. This should suffice to illustrate the qualitative effect of the anomalous dimension on $v_{n}$.

In the $\mathcal{A}=0$ isotropic limit the transverse momentum distribution takes the form

$$
\frac{1}{\pi} \frac{d N}{d k_{T}^{2}}=\frac{1}{4 \pi} \frac{Q_{s}}{\left(k_{T}^{2}+Q_{s}^{2}\right)^{3 / 2}} .
$$

In the presence of a polarized condensate, i.e. for $\mathcal{A}>0$, we consider the limits $k_{T} \gg Q_{s}$ and $k_{T} \ll Q_{s}$ separately. At high transverse momentum we can expand (29) in powers of $r$. The leading contribution $\sim r$, for $\mathcal{A} \ll 1$, gives

$$
\frac{d N}{d^{2} k_{T}}=\left(1-\frac{3 \mathcal{A}}{2}+3 \mathcal{A} \cos ^{2} \phi_{k}\right) \frac{1}{4 \pi} \frac{Q_{s}}{k_{T}^{3}} \quad\left(k_{T} \gg Q_{s}, \mathcal{A} \ll 1\right) .
$$

From this expression we find that

$$
\begin{aligned}
v_{2}\left(k_{T}\right) & =\frac{3}{4} \mathcal{A}\left(k_{T}\right), \\
v_{2 n \geq 4} & =0 .
\end{aligned}
$$

Hence, for $k_{T} \gg Q_{s}$ we find that the anomalous dimension $\gamma$ does not affect $v_{2}\left(k_{T}\right)$ and $v_{4}\left(k_{T}\right)$ qualitatively.

For $k_{T} \ll Q_{s}$ we start by writing the transverse momentum distribution in the form

$$
\frac{d N}{d^{2} k_{T}}=\frac{1}{\pi} \int_{-\pi}^{\pi} d \phi_{r} \frac{1}{\left(Q_{s} \sqrt{1+\mathcal{A} \cos 2 \phi_{r}}+2 i k_{T} \cos \left(\phi_{r}-\phi_{k}\right)\right)^{2}} .
$$

Expanding in powers of $k_{T}$ we find that

$$
\frac{d N}{d^{2} k_{T}}=\frac{1}{Q_{s}^{2}}\left(\frac{2}{\sqrt{1-\mathcal{A}^{2}}}-12 \frac{k_{T}^{2}}{Q_{s}^{2}} \frac{1-\mathcal{A} \cos 2 \phi_{k}}{\left(1-\mathcal{A}^{2}\right)^{3 / 2}}\right) .
$$

Averaging this expression over $\phi_{k}$ gives the normalization factor

$$
\mathcal{N}=\frac{1}{Q_{s}^{2}}\left(\frac{2}{\sqrt{1-\mathcal{A}^{2}}}-12 \frac{k_{T}^{2}}{Q_{s}^{2}} \frac{1}{\left(1-\mathcal{A}^{2}\right)^{3 / 2}}\right)
$$

Thus,

$$
\begin{aligned}
v_{2}\left(k_{T}\right) & =\frac{6}{\mathcal{N}} \frac{k_{T}^{2}}{Q_{s}^{4}} \frac{\mathcal{A}}{\left(1-\mathcal{A}^{2}\right)^{3 / 2}}=\frac{3 \mathcal{A} k_{T}^{2}}{\left(1-\mathcal{A}^{2}\right) Q_{s}^{2}-6 k_{T}^{2}}, \\
v_{4}\left(k_{T}\right) & =0
\end{aligned}
$$

Note that this expression is valid only as long as $\mathcal{N}>0$, i.e. for $k_{T}^{2} \ll\left(1-\mathcal{A}^{2}\right) Q_{s}^{2} / 6$. Hence, for $\gamma=1 / 2$ we find that $v_{4}$ (and all higher harmonics) vanish at low $k_{T}$, in contrast to the classical model where $v_{4} \sim \mathcal{A}^{2} k_{T}^{4}$, eq. (28).

Table I summarizes our results from eqs. $(23,27,28,32,33,37)$.

\begin{tabular}{c|c|c|c}
\hline & $v_{2}$ & $v_{4}$ & $\gamma$ \\
\hline$k_{T} \gg Q_{s}$ & $\mathcal{A}\left(k_{T}\right)$ & 0 & $\gamma \rightarrow 1$ \\
& $\mathcal{A}\left(k_{T}\right)$ & 0 & $\gamma=1 / 2$ \\
\hline$k_{T} \ll Q_{s}$ & $\mathcal{A} k_{T}^{2}$ & $\mathcal{A}^{2} k_{T}^{4}$ & $\gamma \rightarrow 1$ \\
& $\mathcal{A} k_{T}^{2}$ & 0 & $\gamma=1 / 2$ \\
\hline
\end{tabular}

TABLE I: Summary of the leading dependence on $k_{T}$ and condensate amplitude $\mathcal{A}$ of $v_{2}$ and $v_{4}$. 


\section{MODELS FOR THE IMAGINARY PART OF THE DIPOLE SCATTERING AMPLITUDE}

In this section we consider the angular distribution due to the imaginary part of the dipole forward scattering amplitude. We restrict to the semi-classical approximation and take $[8]$

$$
i O(\vec{r}) \sim i \vec{r} \cdot \vec{\nabla}_{b} D(\vec{r}, \vec{b}) .
$$

The proportionality constant is given in ref. [8] and shall be restored later. Here $\vec{b}$ denotes the transverse coordinate of the dipole center of mass (relative to the center of the nucleus): $\vec{b}=(\vec{x}+\vec{y}) / 2$ with $\vec{x}$ and $\vec{y}$ the endpoints of the dipole. $D(\vec{r}, \vec{b})$ depends on $\vec{b}$ through $Q_{s}^{2}(\vec{b})$. Throughout the manuscript we work in the approximation where the nucleus is infinite and homogeneous on average so that the average $Q_{s}^{2}(\vec{b})=Q_{s}^{2}$ is constant. However, the projectile dipole can still couple to short wavelength fluctuations in the target and provide a non-vanishing odderon contribution $O(\vec{r})$. To account for coupling to fluctuations at the scale $r$ we generalize (39) to

$$
i O(\vec{r}) \sim i \int_{\vec{y}}^{\vec{x}} d \vec{s} \cdot \vec{\nabla} D(\vec{r}, \vec{s}) .
$$

This resembles the situation encountered in ref [10] where the dipole S-matrix was computed from the AdS/CFT correspondence: the Nambu-Goto action involves an integration of the (fluctuating) target density along the string connecting the quark and anti-quark. In case that the fluctuations occur on scales larger than $\sim r$, eq. (40) returns to (39) as the average gradient along the dipole can then be taken at the midpoint $\vec{b}$.

We begin by assuming random polarization of electric fields in the target, so $\mathcal{A}=0$ in the notation of the previous section; the effect of the $\vec{E}$ field condensate will be discussed below. In the classical field limit then

$$
i O(\vec{r}) \sim-\frac{i}{4} r^{2} \log \frac{1}{\Lambda r} e^{-\frac{1}{4} r^{2} Q_{s}^{2} \log \frac{1}{\Lambda r}} \int_{\vec{y}}^{\vec{x}} d \vec{s} \cdot \vec{\nabla} Q_{s}^{2}(\vec{s}) .
$$

If the fluctuation in the target is dominated by a single mode we may write

$$
\begin{aligned}
\frac{Q_{s}^{2}(\vec{s})}{Q_{s}^{2}} & =1+\int \frac{d^{2} q}{(2 \pi)^{2}} \delta f(\vec{q}) e^{i \vec{q} \cdot \vec{s}} \\
\delta f(\vec{q}) & =\frac{(2 \pi)^{2}}{2} \mathcal{B}\left(q_{0}\right)\left[(1+i) \delta\left(\vec{q}-\vec{q}_{0}\right)+(1-i) \delta\left(\vec{q}+\vec{q}_{0}\right)\right] .
\end{aligned}
$$

Here, $\vec{q}_{0}=\left(q_{0}, 0\right)$ determines the scale of the fluctuation and the direction for spontaneous breaking of rotational symmetry. $\mathcal{B}\left(q_{0}\right)$ is the amplitude of the fluctuation. Note that the fluctuation satisfies $\delta f(\vec{q})=\delta f^{*}(-\vec{q})$ so that $Q_{s}^{2}(\vec{s})$ is real. We should stress that the direction of the wave vector $\vec{q}$ of the fluctuation would, in principle, be arbitrary and that it need not coincide with the direction of the condensate which generates the even harmonics. However, here we shall not be concerned with correlations of the "event planes" of $v_{n}$ and so we fix $\vec{q}=\left(q_{0}, 0\right)$ to point in the same direction as the $\vec{E}$ field condensate discussed in the previous section.

With this ansatz,

$$
\begin{aligned}
\int_{\vec{y}}^{\vec{x}} d \vec{s} \cdot \vec{\nabla} Q_{s}^{2}(\vec{s}) & =-Q_{s}^{2} \mathcal{B}\left(q_{0}\right) \int_{\vec{y}}^{\vec{x}} d \vec{s} \cdot \vec{q}_{0}\left[\sin \left(\vec{q}_{0} \cdot \vec{s}\right)+\cos \left(\vec{q}_{0} \cdot \vec{s}\right)\right] \\
& =-2 Q_{s}^{2} \mathcal{B}\left(q_{0}\right) \sin \frac{\overrightarrow{q_{0}} \cdot \vec{r}}{2}
\end{aligned}
$$

where we parametrized the line from $\vec{y}$ to $\vec{x}$ as $\vec{s}(\sigma)=\vec{b}+\vec{r} \sigma$ with $\sigma \in\left[-\frac{1}{2}, \frac{1}{2}\right]$, and then set $\vec{b}=0$.

Hence, we obtain

$$
i O(\vec{r}) \sim \frac{i}{2} r^{2} Q_{s}^{2} \mathcal{B}\left(q_{0}\right) \sin \left(\frac{1}{2} r q_{0} \cos \phi_{r}\right) \log \frac{1}{\Lambda r} e^{-\frac{1}{4} r^{2} Q_{s}^{2} \log \frac{1}{\Lambda r}}
$$


To obtain some qualitative insight consider the limit of high transverse momentum resp. $r \ll 1 / Q_{s}$. In this regime we can drop the exponential in (46):

$$
i O(\vec{r}) \sim \frac{i}{2} r^{2} Q_{s}^{2} \mathcal{B}\left(q_{0}\right) \sin \left(\frac{1}{2} r q_{0} \cos \phi_{r}\right) \log \frac{1}{\Lambda r} \quad\left(r Q_{s} \ll 1\right) .
$$

A very long wavelength fluctuation (as compared to the size of the dipole) corresponds to $r q_{0} \ll 1$ and the leading approximation for the sine leads to

$$
\frac{d N}{d^{2} k_{T}} \sim \mathcal{B}\left(q_{0}\right) \frac{q_{0}}{k_{T}} \frac{Q_{s}^{2}}{k_{T}^{4}} \cos \left(\phi_{k}\right)
$$

In fact, the limit $r q_{0} \ll 1$ corresponds to evaluating the gradient of $D(\vec{x}, \vec{y})$ at the midpoint $\vec{b}$ as in eq. (39). This angular distribution evidently corresponds to $v_{1}\left(k_{T}\right) \neq 0$ but $v_{3}\left(k_{T}\right)=0$.

Fluctuations at the scale $r q_{0} \gtrsim 1$ do lead to higher harmonics, however. To show that we integrate eq. (46) over a scale invariant spectrum of fluctuations:

$$
\mathcal{B}\left(q_{0}\right) \rightarrow \frac{1}{2} \mathcal{B} \int \frac{d q_{0}^{2}}{q_{0}^{2}}
$$

acting on the sine function in that expression. Note that in doing so, we are still restricting to the somewhat extreme case where all wave vectors $\vec{q}_{0}$ are pointing in the same direction; some angular smearing of the fluctuation vectors about the $\vec{x}$-direction could be incorporated in the future. This turns eq. (46) into

$$
i O(\vec{r}) \sim \frac{i \pi}{4} r^{2} Q_{s}^{2} \mathcal{B} \operatorname{sgn}\left(\cos \phi_{r}\right) \log \frac{1}{\Lambda r} e^{-\frac{1}{4} r^{2} Q_{s}^{2} \log \frac{1}{\Lambda r}} .
$$

We can generalize this equation by adding an exponential fluctuation cutoff beyond a scale $Q_{c}$ :

$$
\mathcal{B}\left(q_{0}\right) \rightarrow \frac{1}{2} \mathcal{B} \int \frac{d q_{0}^{2}}{q_{0}^{2}} e^{-q_{0} / Q_{c}}
$$

which again is acting on the sine function in eq. (46). Then the C-odd part of the S-matrix is

$$
i O(\vec{r}) \sim \frac{i}{2} r^{2} Q_{s}^{2} \mathcal{B} \arctan \left(\frac{1}{2} r Q_{c} \cos \phi_{r}\right) \log \frac{1}{\Lambda r} e^{-\frac{1}{4} r^{2} Q_{s}^{2} \log \frac{1}{\Lambda r}} .
$$

Eq. (50) is reproduced when $Q_{c} \rightarrow \infty$. The expansion for small dipoles, $r^{-1} \gg Q_{c}, Q_{s}$ is

$$
i O(\vec{r}) \sim i r^{3} Q_{s}^{2} Q_{c} \mathcal{B} \cos \phi_{r}\left[1-\frac{r^{2}}{4}\left(\frac{Q_{c}^{2} \cos ^{2} \phi_{r}}{3}+Q_{s}^{2}\right)\right]
$$

up to logarithms of $1 / r$. Hence, $v_{1}$ is generated already at order $r^{3}$ while $v_{3}$ only appears at order $r^{5}$ and would therefore drop by an additional $1 / k_{T}^{2}$ at high transverse momentum.

Finally, we restore the proper prefactor of $i O(\vec{r})$ as given in ref. [8]:

$$
i O(\vec{r})=i \frac{\alpha_{s}\left(N_{c}^{2}-4\right)}{2^{6}} \mathcal{B} \arctan \left(\frac{1}{2} r Q_{c} \cos \phi_{r}\right) \rho^{2} e^{-\rho^{2}},
$$

with

$$
\rho^{2}=\frac{1}{4} r^{2} Q_{s}^{2} \log \frac{1}{\Lambda r}
$$

The effect of the $\vec{E}$ field condensate would be to modify this expression to

$$
\rho_{\mathcal{A}}^{2}=\rho^{2}\left(1-\mathcal{A}+2 \mathcal{A} \cos ^{2} \phi_{r}\right)
$$

Eq. (56) assumes that the fluctuations which generate odd harmonics are perfectly aligned with the $\vec{E}$ field condensate which generates even $v_{2 n}$. For simplicity, we shall assume that this is not the case and instead take the event planes of $v_{2 n}$ and $v_{2 n+1}$ to have random relative orientations. Thus, below we shall use eq. (55). 


\section{NUMERICAL RESULTS}

In this section we present numerical results for the azimuthal harmonics $v_{n}$. We have discretized $\vec{r}$-space on a two-dimensional cartesian lattice, performed a Fourier transform to $2 d$ discrete $\vec{k}$-space where we then evaluated

$$
v_{n}(k)=e^{i \pi \delta_{n 1}}\left(2 \frac{d N}{d k^{2}}\right)^{-1} \int d \phi_{k} \frac{d N}{d^{2} k} \cos n \phi_{k} .
$$

Even if $d N / d^{2} k$ is chosen to be symmetric under $\phi_{k} \rightarrow-\phi_{k}$ there is, in general, an undetermined phase $\exp (i \pi j)$ with integer $j$ which is set by convention. The additional minus sign in the definition of $v_{1}$ is due to the convention that $v_{1}\left(p_{T}\right)<0$ at low $p_{T}$ crossing over to $v_{1}\left(p_{T}\right)>0$ at high $p_{T}$.

In the numerical evaluation of $d N / d^{2} k$ we assume $Q_{s} / \Lambda=10$ so that any dependence on the infrared cutoff $\Lambda$ should be weak. Also, we made sure that the lattice dimension $L Q_{s} \sim 250$ far exceeds the relevant physical scale in the problem. Lastly, in all cases the dimensionless lattice saturation scale $Q_{s}^{L} \equiv a Q_{s} \ll 1$ (where $a$ denotes the lattice spacing in $r$-space) so that discretization effects should be small. In fact, we performed a continuum extrapolation taking $Q_{s}^{L} \rightarrow 0$ at fixed $L Q_{s}$.

The transverse momentum distribution $d N / d^{2} k$ is obtained by a Fourier transform of the dipole S-matrix as written in eq. (8). We employ the semi-classical model for the S-matrix given by

$$
\operatorname{Re} S(\vec{r}) \equiv D(\vec{r})=e^{-\rho^{2}\left(1-\mathcal{A}+2 \mathcal{A} \cos ^{2} \phi_{r}\right)},
$$

and

$$
\operatorname{Im} S(\vec{r}) \equiv O(\vec{r})=\frac{\alpha_{s}\left(N_{c}^{2}-4\right)}{64} \mathcal{B} \arctan \left(\frac{1}{2} r Q_{c} \cos \phi_{r}\right) \rho^{2} e^{-\rho^{2}}
$$

with

$$
\rho^{2}=\frac{1}{4} r^{2} Q_{s}^{2} \log \left(e+\frac{1}{\Lambda r}\right) .
$$

Here, we have added a constant to the argument of the logarithm to avoid an unphysical sign flip in the deep infrared. Below, we restrict to dense targets $Q_{s} \gg \Lambda$ where the regime $r>1 / \Lambda$ plays no crucial role.

We repeat that, in principle, in eq. (59) $\phi_{r} \rightarrow \phi_{r}+\psi_{0}$ could be shifted by a random "event plane angle", which is of no consequence for our analysis. Also, we shall take $\alpha_{s}=0.25, N_{c}=3$, and consider the $\vec{E}$-field polarization amplitude $\mathcal{A}$ and the strength of fluctuations $\mathcal{B}$ as free parameters.

The main goal of this section is to show a few main qualitative features of the equations presented above. We do not yet attempt a quantitative description of the data. We therefore restrict to angular harmonics for elastic quark scattering and omit the convolutions with quark $\rightarrow$ hadron fragmentation and with the proton parton distribution functions. Nevertheless, for a rough comparison to the data we rescale the transverse momentum as $p_{T}=k_{T} / 2$.

\section{A. Even harmonics}

In fig. 1 we show $v_{2}\left(p_{T}\right)$ from the semi-classical dipole model. In this plot we have scaled the experimental $p_{T}$ by $Q_{s}=1.3 \mathrm{GeV}$; note that this numerical value is strongly correlated to $\langle z\rangle=1 / 2$ assumed in our rescaling of quark momenta. To extract a more physical value for $Q_{s}$ one needs to account for the additional convolutions with distribution and fragmentation functions which we postpone to future work.

Despite the qualitative nature of our numerical results we may note that with an $\vec{E}$-field polarization amplitude of $\mathcal{A}=7 \%$ the model reproduces the magnitude and the rough $p_{T}$ dependence of the data. Our analysis provides a first order of magnitude estimate of $\mathcal{A}$ which was previously unknown [9]. Also, with this value for $\mathcal{A}$ we find that $v_{4}\left(p_{T}\right) \leq 1 \%$ is very small ${ }^{5}$, as expected from the analytical discussion in section IV A.

In fig. 1 we have (somewhat arbitrarily) focused on the highest multiplicity $\mathrm{p}+\mathrm{Pb}$ events analyzed by CMS. A study of the multiplicity dependence of $v_{n}\left(p_{T}\right)$ is beyond the scope of this work. Nevertheless, it appears that the more rapid drop of $v_{2}\left(p_{T}\right)$ (from four particle correlations) at high $p_{T}$ in events with lower multiplicity may require a scale dependent polarization strength $\mathcal{A}$. We intend to return to this question in the future.

\footnotetext{
${ }^{5}$ We repeat though that here we have not performed an average over multiple $\vec{E}$-field domains explicitly, see next section.
} 


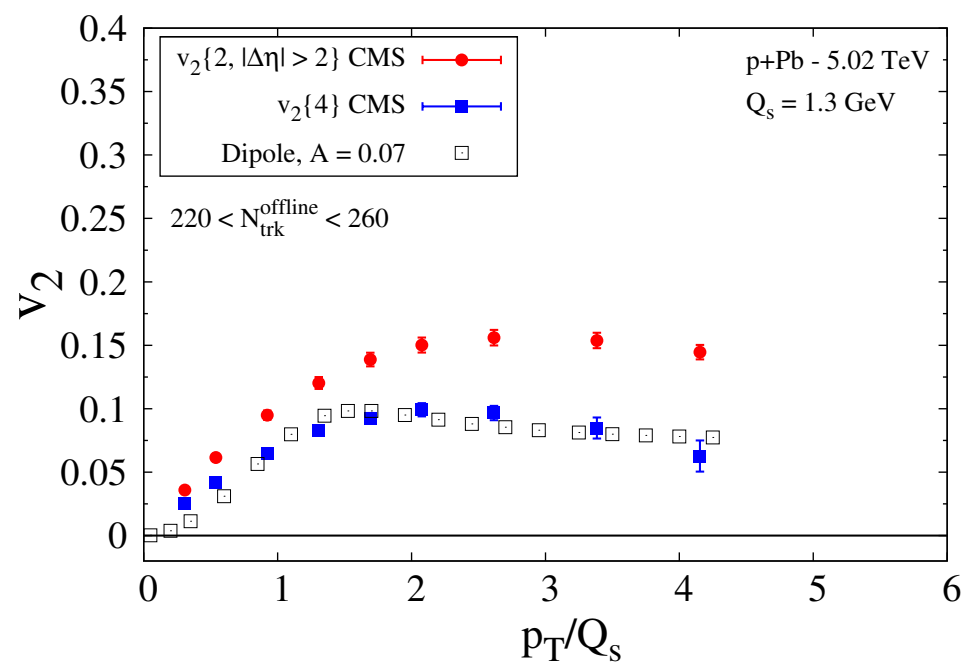

FIG. 1: $v_{2}\left(p_{T}\right)$ from the semi-classical dipole model with $\vec{E}$-field polarization amplitude $\mathcal{A}=7 \%$ is shown by open squares. Data by the CMS collaboration is shown as filled circles $\left(v_{2}\{2\}\right)$ and filled squares $\left(v_{2}\{4\}\right)$, respectively, and corresponds to very high multiplicity $\mathrm{p}+\mathrm{Pb}$ collisions at $\sqrt{ } s=5.02 \mathrm{TeV}$.

\section{B. Domain model}

In the previous section we considered a single target domain and determined the effective polarization at $\mathcal{A}=7 \%$. This relatively small value of $\mathcal{A}$ may be due to the fact that we are in effect averaging over multiple domains where $\vec{E}$ points in different directions. This prevented us from making a prediction for the "quadrangular asymmetry" $v_{4}$ which may average differently than $v_{2}$. These questions can be addressed in a simple domain model.

One first introduces $m$-particle cumulants [12], as already mentioned in the introduction. For example, the twoparticle correlation is defined as

$$
v_{n}^{2}\{2\} e^{i \psi} \equiv\left\langle e^{i n\left(\phi_{1}-\phi_{2}\right)}\right\rangle=\frac{1}{\mathcal{N}} \int \frac{d \phi_{1}}{2 \pi} \frac{d \phi_{2}}{2 \pi} e^{i n\left(\phi_{1}-\phi_{2}\right)} \frac{d N}{d \phi_{1} d^{2} k_{1}} \frac{d N}{d \phi_{2} d^{2} k_{2}}+\cdots
$$

with an obvious generalization to $m>2$ particles. The phase $\psi$ is not important here. On the right, we have assumed that the two-particle angular distribution factorizes into a product of two single-particle distributions, plus corrections. Thus, if both particles are produced from the same target domain, we obtain that $v_{n}\{2\}=v_{n}$ as computed above. On the other hand, if (at least) one of the particles is produced from an uncorrelated domain (with $\vec{E}$ pointing in a random direction) then $v_{n}\{2\}=0$. In all, for $N_{D}$ domains we find that $v_{n}$ from $m$-particle correlations is given by

$$
v_{n}\{m\}=v_{n} N_{D}^{\frac{1-m}{m}} .
$$

Fig. 2 shows our fit to $v_{2}\{4\}$ with larger target field polarization $\mathcal{A}=0.15,0.20,0.25$; the overall magnitude has been adjusted via $N_{D}$. At the same time, we attempt to "saturate" as much as possible, but not to overshoot the measured two-particle correlation $v_{2}\{2\}^{6}$.

We find that the simple domain model fits best with $\mathcal{A} \simeq 0.20$ and $N_{D} \simeq 4.3$ : larger values for $\mathcal{A}$ require larger $N_{D}$ which in turn results in too strong splitting of $v_{2}\{2\}$ and $v_{2}\{4\}$ and is not compatible with the data. In this model there appears to be some room for genuine (non-factorizable) two-particle correlations at high $p_{T}$.

Having determined $\mathcal{A}$ and $N_{D}$ we can now use the same model for $v_{4}\{2\}$ and $v_{4}\{4\}$. The former has been measured by the ATLAS collaboration and provides another test of the domain model. As shown in fig. 3, the model does fit $v_{4}\{2\}$ about $Q_{s}$ but underestimates it at higher $p_{T}$. This may be interpreted as due to the presence of non-factorizable correlations which are not accounted for.

\footnotetext{
${ }^{6}$ We do not require a perfect description of $v_{2}\{2\}$ over the entire range of $p_{T}$ since our present analysis neglects genuine two-particle
} correlations, as indicated by the dots in eq. (61). 

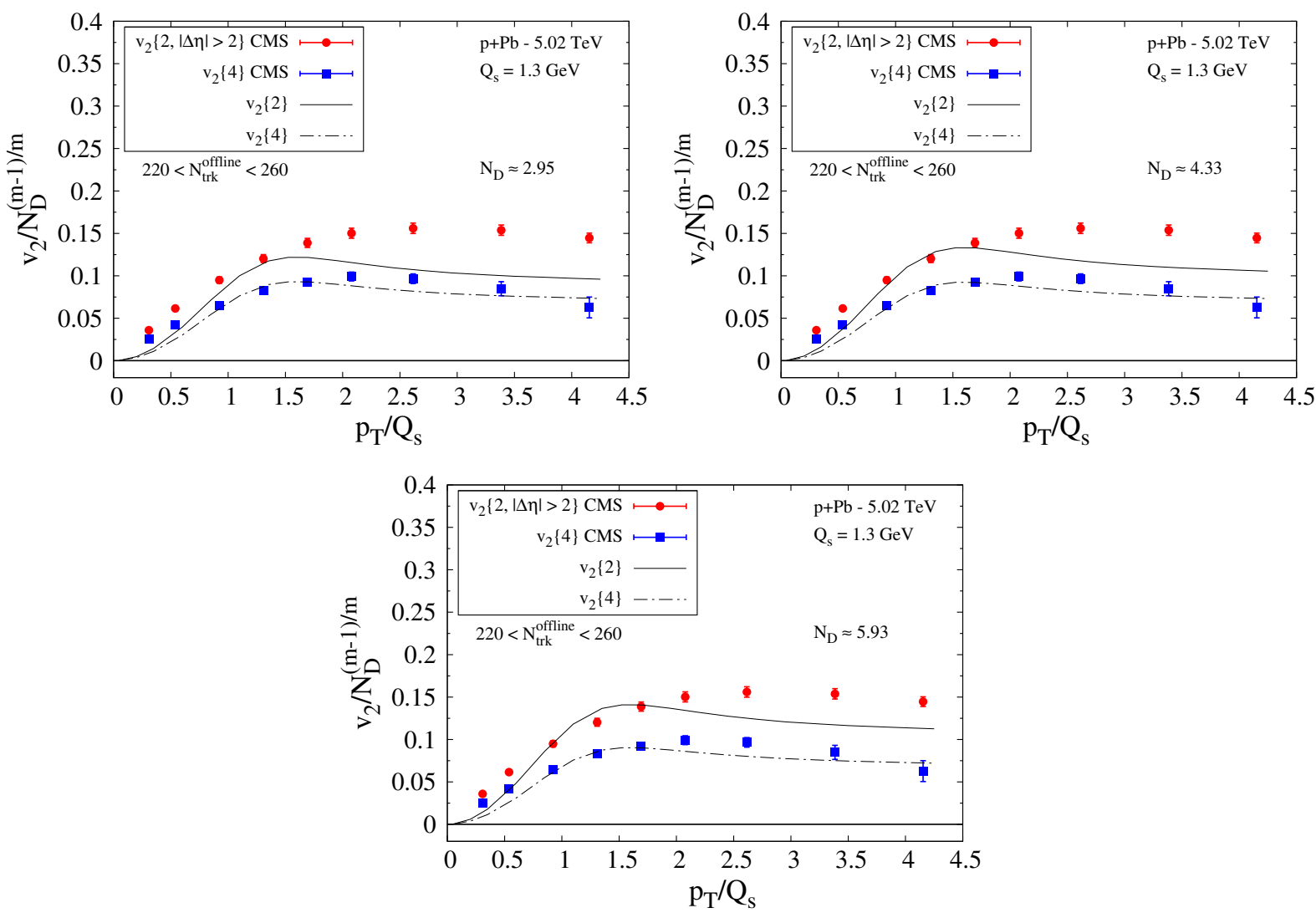

FIG. 2: $v_{2}\left(p_{T}\right)$ from two- and four-particle cumulants. The domain model with $N_{D}=2.95,4.33$, 5.93 (and, correspondingly, $\mathcal{A}=0.15,0.20,0.25)$ is compared to data by the CMS collaboration.

The prediction for $v_{4}\{4\}$ is shown in fig. 3 , right. The model predicts a rather small $v_{4}\{4\} \lesssim 2 \%$ over the entire range of $p_{T}$. More importantly, while $v_{2}\{4\}$ above $Q_{s}$ is rather flat, $v_{4}\{4\}$ clearly decreases with $p_{T}$. This is a rather generic prediction of the $\sim \vec{r} \cdot \vec{E}$ dipole interaction with the vector electric field.

\section{Odd harmonics}

We now turn to odd harmonics. The $p_{T}$ dependence of $v_{1}$ and $v_{3}$ for different cutoffs $Q_{c}$ is shown in fig. 4. For this figure the amplitude of C-odd fluctuations has been fixed to $\mathcal{B}=1$ as it only affects the magnitude but not the $p_{T}$ dependence of the angular moments. Also, we keep $\mathcal{A}=7 \%$ from above and again rescale from quark to hadron momenta as $p_{T}=k_{T} / 2$.

We observe that for $p_{T} / Q_{s} \lesssim 2, v_{1}\left(p_{T}\right)$ depends rather weakly on the cutoff $Q_{c}$; at high $p_{T}$ it increases somewhat with $Q_{c}$ until the cutoff far exceeds the saturation scale. On the other hand, $v_{3}$ at high $p_{T}$ increases rather rapidly with $Q_{c}$. This behavior is in line with the analytic discussion in the previous section: fluctuations with wave length on the order of the dipole size $r$ only produce $v_{1}$ but not $v_{3}$.

We can now "tune" the fluctuation amplitude $\mathcal{B}$ and the cutoff $Q_{c}$ to ATLAS data for $v_{1}\left(p_{T}\right)$ from high multiplicity $\mathrm{p}+\mathrm{Pb}$ collisions at $5 \mathrm{TeV}$, and then check the behavior of $v_{3}\left(p_{T}\right)$ against the data from CMS. We should, of course, keep in mind that the data corresponds to two-particle correlations $v_{1,3}\{2\}$ while the model gives $\left\langle\cos n \phi_{k}\right\rangle$ evaluated on the single-inclusive particle distribution, averaged over events.

In fig. 5 we show that the overall magnitude of both $v_{1}$ and $v_{3}$ is reproduced by the odderon model with a fluctuation amplitude of $\mathcal{B}=1.6$; we remind the reader that this amplitude effectively incorporates the contribution of gluons to the denominator in eq. (57).

Furthermore, a cutoff $Q_{c} \sim 18 Q_{s}$ leads to a decent description of both $v_{1}$ and $v_{3}$ at transverse momenta of a few times $Q_{s}$. Of course, our model provides no deeper explanation for the presence of such hard fluctuations and it will 

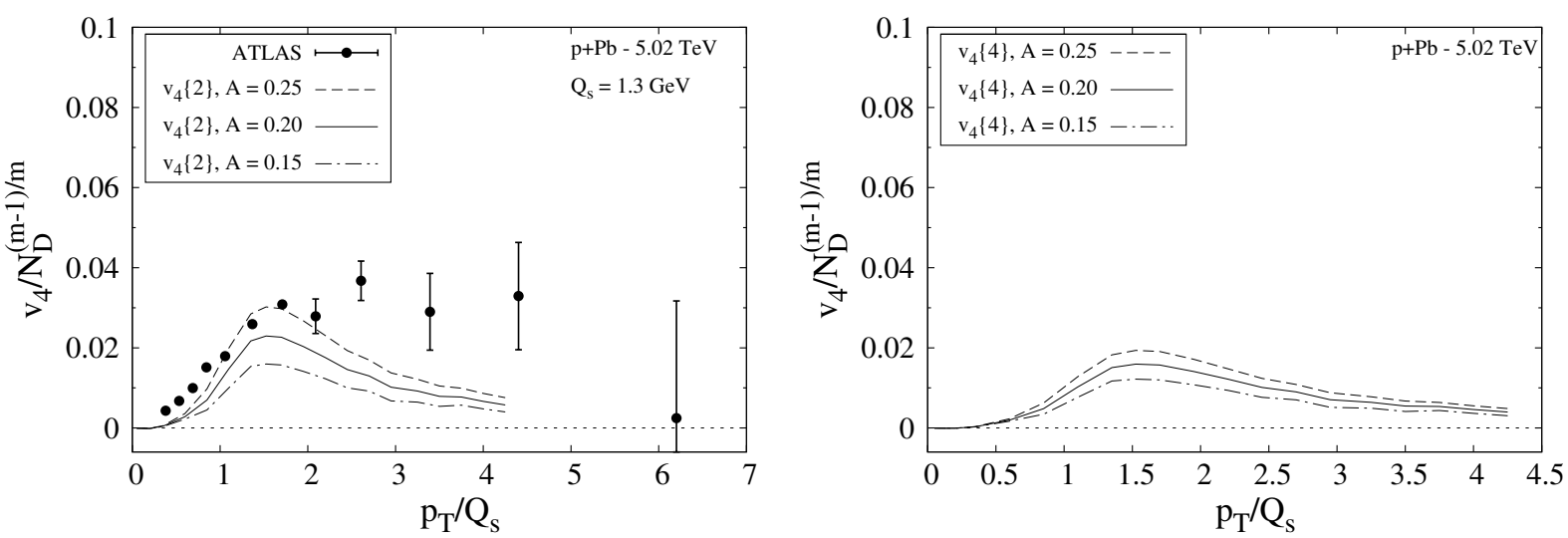

FIG. 3: $v_{4}\left(p_{T}\right)$ from two- and four-particle cumulants. On the left, the domain model with $N_{D}=2.95,4.33,5.93$ (and, correspondingly, $\mathcal{A}=0.15,0.20,0.25$; from bottom to top) is compared to $v_{4}\{2\}$ data by the ATLAS collaboration. On the right we plot the model prediction for $v_{4}\{4\}$ for the same combinations of $\mathcal{A}$ and $N_{D}$.
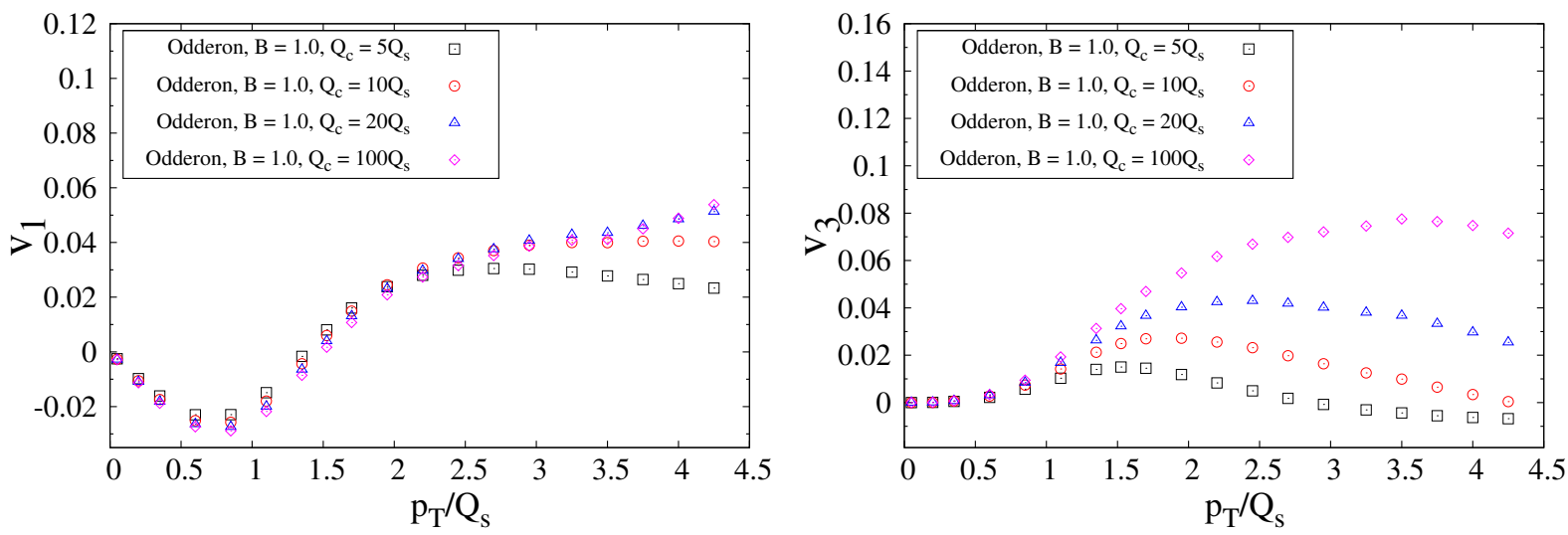

FIG. 4: $v_{1}\left(p_{T}\right)$ and $v_{3}\left(p_{T}\right)$ from the semi-classical odderon model for a scale invariant distribution of C-odd fluctuations with a cutoff $Q_{c}$.

be important to understand this result in more detail in the future (perhaps from the Liouville effective theory for fluctuations of $Q_{s}$ proposed in ref. [28]). Nevertheless, it is quite interesting that the continued rise of $v_{1}$ at $p_{T} / Q s \gtrsim 2$, together with the drop of $v_{3}$ at such $p_{T}$, is basically reproduced by the odderon model (59) in the presence of very short wavelength fluctuations in the target.

\section{SUMMARY AND OUTLOOK}

In this paper we have provided a first qualitative analysis of azimuthal asymmetries within the dipole approach. Following earlier work by Kovner and Lublinsky [9] we have introduced a semi-classical dipole scattering amplitude which accounts for spontaneous breaking of 2D rotational symmetry due to a condensate for the (color) electric field $\vec{E}$. Our numerical estimates indicate that this model is able to describe the $p_{T}$-dependence of the elliptic asymmetry $v_{2}$ obtained from four-particle correlations (or from a global event plane method).

The second goal of this paper was to address parity odd azimuthal moments $v_{2 n+1}$. These arise from the C-odd imaginary part of the dipole S-matrix, analogous to single transverse spin asymmetries discussed before by Kovchegov and Sievert [8]. However, in order to obtain non-zero $v_{3}$ we have found it necessary to generalize the odderon to a string-like operator [10] so that the dipole can couple to hard fluctuations of wave length below the dipole size $r \sim 1 / p_{T}$. Fixing two parameters, the fluctuation amplitude $\mathcal{B}$ and cutoff $Q_{c}$, we find that the odderon model provides a reasonably good simultaneous description of $v_{1}\{2\}\left(p_{T}\right)$ and $v_{3}\{2\}\left(p_{T}\right)$ measured in high multiplicity $\mathrm{p}+\mathrm{Pb}$ collisions at the LHC. We should emphasize, however, that it would probably be more appropriate to compare to 

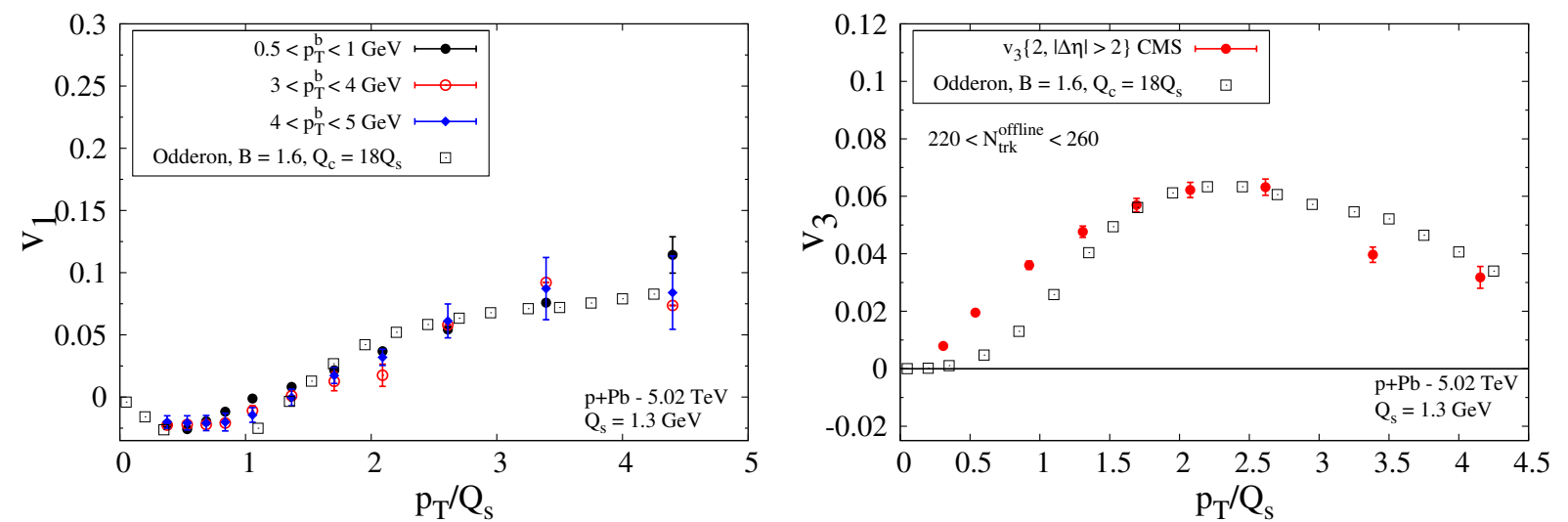

FIG. 5: $v_{1}\left(p_{T}\right)$ and $v_{3}\left(p_{T}\right)$ from the semi-classical odderon model with fluctuation amplitude $\mathcal{B}=1.6$ and cutoff $Q_{c} / Q_{s}=18$. Data by the ATLAS $\left(v_{1}\right)$ and CMS $\left(v_{3}\right)$ collaborations for $v_{1,3}\{2\}$ corresponds to very high multiplicity $\mathrm{p}+\mathrm{Pb}$ collisions at $\sqrt{ } s=5.02 \mathrm{TeV}$.

$v_{1}\left(p_{T}\right)$ and $v_{3}\left(p_{T}\right)$ obtained from four or more particle correlations (or from a global event plane method), which is not currently available.

As already alluded to above, the main intention of the present work was to explore some qualitative features of the underlying ideas. Much work remains to be done before a more quantitative comparison to data could be attempted. For example, we have not at all addressed the nature of the high-multiplicity $\mathrm{p}+\mathrm{Pb}$ events which display (large) azimuthal anisotropies. Also, it will be important to employ better models for the dipole $D(\vec{r})$ and odderon $O(\vec{r})$. Finally, we will have to develop some theoretical understanding for the amplitude and cutoff of fluctuations, which here we extracted phenomenologically.

In closing, we should point out that other possible explanations for the observed azimuthal asymmetries in $\mathrm{p}+\mathrm{A}$ collisions are presently under intense investigation. For example, initial density inhomogeneities of the "fireball" in the transverse plane could be converted into momentum space asymmetries in the final state by hydrodynamic flow [29]. Hydrodynamics is an effective theory describing the propagation of long wavelength density perturbations. Our present approach is complementary and relies on short distance physics corresponding to transverse momenta far beyond $\sim \Lambda_{\mathrm{QCD}}$. In the future, it will be important to understand at what scale $p_{T}$ one transitions from one description to the other.

\section{Acknowledgements}

We thank K. Dusling, V. Skokov, and R. Venugopalan for useful discussions. A.D. gratefully acknowledges support by the DOE Office of Nuclear Physics through Grant No. DE-FG02-09ER41620 and from The City University of New York through the PSC-CUNY Research Award Program, grant 66514-0044. A.V.G. gratefully acknowledges the Brazilian Funding Agency FAPESP for financial support (contract: 2013/23848-5).

[1] B. Abelev et al. [ALICE Collaboration], Phys. Lett. B 719, 29 (2013); arXiv:1406.2474 [nucl-ex].

[2] G. Aad et al. [ATLAS Collaboration], Phys. Rev. Lett. 110, 182302 (2013); Phys. Lett. B 725, 60 (2013).

[3] The ATLAS collaboration, ATLAS-CONF-2014-021.

[4] S. Chatrchyan et al. [CMS Collaboration], Phys. Lett. B 718, 795 (2013); Phys. Lett. B 724, 213 (2013).

[5] A. Adare et al. [PHENIX Collaboration], Phys. Rev. Lett. 111, 212301 (2013).

[6] A. Dumitru, F. Gelis, L. McLerran and R. Venugopalan, Nucl. Phys. A 810, 91 (2008).

[7] A. Adare et al. [PHENIX Collaboration], arXiv:1404.7461 [nucl-ex].

[8] Y. V. Kovchegov and M. D. Sievert, Phys. Rev. D 86, 034028 (2012) [Erratum-ibid. D 86, 079906 (2012)].

[9] A. Kovner and M. Lublinsky, Phys. Rev. D 84, 094011 (2011).

[10] J. Noronha and A. Dumitru, Phys. Rev. D 89, 094008 (2014).

[11] Y. V. Kovchegov and K. L. Tuchin, Nucl. Phys. A 708, 413 (2002).

[12] N. Borghini, P. M. Dinh and J. -Y. Ollitrault, Phys. Rev. C 64, 054901 (2001).

[13] M. Gyulassy, P. Levai, I. Vitev and T. Biro, arXiv:1405.7825 [hep-ph]. 
[14] A. Bzdak, P. Bozek and L. McLerran, arXiv:1311.7325 [hep-ph]; L. Yan and J.-Y. Ollitrault, Phys. Rev. Lett. 112, 082301 (2014); A. Bzdak and V. Skokov, arXiv:1312.7349 [hep-ph].

[15] A. Kovner and M. Lublinsky, Phys. Rev. D 83, 034017 (2011).

[16] A. Dumitru, K. Dusling, F. Gelis, J. Jalilian-Marian, T. Lappi and R. Venugopalan, Phys. Lett. B 697, 21 (2011); K. Dusling and R. Venugopalan, Phys. Rev. Lett. 108, 262001 (2012); Phys. Rev. D 87, 054014 (2013); Phys. Rev. D 87, 094034 (2013).

[17] B. Alver and G. Roland, Phys. Rev. C 81, 054905 (2010) [Erratum-ibid. C 82, 039903 (2010)].

[18] T. Altinoluk, N. Armesto, G. Beuf, M. Martinez and C. A. Salgado, arXiv:1404.2219 [hep-ph].

[19] J. D. Bjorken, J. B. Kogut and D. E. Soper, Phys. Rev. D 3, 1382 (1971).

[20] A. Dumitru and J. Jalilian-Marian, Phys. Rev. Lett. 89, 022301 (2002).

[21] A. H. Mueller, Nucl. Phys. B 415, 373 (1994); Nucl. Phys. B 437, 107 (1995); A. H. Mueller and B. Patel, Nucl. Phys. B 425, 471 (1994).

[22] A. Dumitru, A. Hayashigaki and J. Jalilian-Marian, Nucl. Phys. A 765, 464 (2006).

[23] T. Altinoluk and A. Kovner, Phys. Rev. D 83, 105004 (2011).

[24] G. A. Chirilli, B.-W. Xiao and F. Yuan, Phys. Rev. Lett. 108, 122301 (2012); Phys. Rev. D 86, 054005 (2012).

[25] C. Andrés, A. Moscoso and C. Pajares, arXiv:1405.3632 [hep-ph].

[26] Y. V. Kovchegov and A. H. Mueller, Nucl. Phys. B 529, 451 (1998).

[27] A. Dumitru, T. Lappi and L. McLerran, Nucl. Phys. A 922, 140 (2014).

[28] E. Iancu and L. McLerran, Nucl. Phys. A 793, 96 (2007).

[29] P. Bozek, Phys. Rev. C 85, 014911 (2012); P. Bozek and W. Broniowski, Phys. Lett. B 718, 1557 (2013); Phys. Rev. C 88, 014903 (2013); A. Bzdak, B. Schenke, P. Tribedy and R. Venugopalan, Phys. Rev. C 87, 064906 (2013); E. Shuryak and I. Zahed, Phys. Rev. C 88, 044915 (2013); P. Bozek, W. Broniowski and G. Torrieri, Phys. Rev. Lett. 111, 172303 (2013); K. Werner, M. Bleicher, B. Guiot, I. Karpenko and T. Pierog, Phys. Rev. Lett. 112, 232301 (2014); B. Schenke and R. Venugopalan, arXiv:1405.3605 [nucl-th]. 